\title{
Impact of multichannel and multipole effects on the Cooper minimum in the high-order-harmonic spectrum of argon
}

\author{
Stefan Pabst, ${ }^{1,2}$ Loren Greenman, ${ }^{3,{ }^{*}}$ David A. Mazziotti, ${ }^{3}$ and Robin Santra ${ }^{1,2, \dagger}$ \\ ${ }^{1}$ Center for Free-Electron Laser Science, DESY, Notkestrasse 85, D-22607 Hamburg, Germany \\ ${ }^{2}$ Department of Physics,University of Hamburg, Jungiusstrasse 9, D-20355 Hamburg, Germany \\ ${ }^{3}$ Department of Chemistry and The James Franck Institute, The University of Chicago, Chicago, Illinois 60637, USA
}

(Received 19 October 2011; published 21 February 2012)

\begin{abstract}
We investigate the relevance of multiple-orbital and multipole effects during high-harmonic generation (HHG). The time-dependent configuration interaction singles (TDCIS) approach is used to study the impact of the detailed description of the residual electron-ion interaction on the HHG spectrum. We find that the shape and position of the Cooper minimum in the HHG spectrum of argon changes significantly whether or not interchannel interactions are taken into account. The HHG yield can be underestimated by up to 2 orders of magnitude in the energy region of 30-50 eV. We show that the argument of low ionization probability is not sufficient to justify ignoring multiple-orbital contributions. Additionally, we find the HHG yield is sensitive to the nonspherical multipole character of the electron-ion interaction.
\end{abstract}

DOI: 10.1103/PhysRevA.85.023411

PACS number(s): $32.80 . \mathrm{Rm}, 42.65 . \mathrm{Re}, 31.15 . \mathrm{A}-$

\section{INTRODUCTION}

High-harmonic generation (HHG) is the key physical process underlying the generation of single attosecond pulses [1-3] and attosecond pulse trains [4-6], which are at the heart of attosecond science [7,8]. In recent years, the rapid progress in HHG has led to applications ranging from atomic systems [9-11] over molecular systems [12,13] to solid-state systems [14]. HHG has opened a new door to probe structural information $[12,15]$ as well as electronic and nuclear dynamics [16-19] on fundamental time scales.

The mechanism behind HHG is well captured in the three-step model [20], where in the first step the electron is tunnel-ionized by a strong-field laser pulse, in the second step the electron is accelerated in the oscillating laser field, and finally in the last step the electron recollides with the parent ion and converts its excess energy into radiation energy in the extreme ultraviolet range $[21,22]$. The maximum photon energy is given by the cutoff law, $1.32 I_{p}+3.17 U_{p}$, where $I_{p}$ is the ionization potential of the system and $U_{p}$ is the ponderomotive potential created by the intense laser field [20]. As a result, the heavier noble-gas atoms have lower cutoff energies than the lighter ones, whereas the HHG yield does increase with the atomic number $[23,24]$. Previous works have shown that the recombination step can be directly related to photoionization [25,26], enabling the retrieval of the electronic structure of the system $[12,25,27,28]$. A strong focus has been, in particular, on molecular systems [29-32].

The two most common theoretical approaches for describing HHG are the semiclassical strong-field approximation (SFA) [20], which has been extended to include Coulombinteraction corrections [25,33-35], and the single-activeelectron (SAE) approximation [36-39], where the electron-ion interaction for many-electron systems is described by a model

\footnotetext{
*Present address: Department of Chemistry, Berkeley Center for Quantum Information and Computation, University of California, Berkeley, CA 94720, USA.

†robin.santra@cfel.de
}

potential [37]. The SAE approach is computationally more demanding than the SFA approach, and, therefore, has been limited to atoms and systems like $\mathrm{H}_{2}^{+}$. In the literature [25], the SAE approximation has often been referred to as solving the time-dependent Schrödinger equation (TDSE). The SAE approach has some limitations. For example, it ignores contributions from multiple orbitals. Intensive studies have been performed to understand the impact of multiple-orbital contributions in molecular systems, which are essential to understand the HHG spectrum and subsequently to extract electronic-structure information [40,41]. Recently, it has been shown that even in atomic systems it is crucial to consider multiple-orbital effects [42].

In this paper, we investigate the importance of multipleorbital (multichannel) contributions and multipole effects in the residual electron-ion interaction on the Cooper minimum in the HHG spectrum of argon $[37,43,44]$. Both aspects are commonly ignored in SFA and SAE calculations. Multichannel interactions [45] go beyond the independent-particle picture and cannot be captured in the language of SFA and SAE, whereas multipole effects could, in principle, arise even in a single-channel model such as SAE. However, in atomic SAE calculations, it is common to model the electron-ion interaction by a spherically symmetric potential [37]. The interaction of the liberated electron with the hole state (channel), from which it originates, is called intrachannel interaction and leads for large electron-ion distances to the $1 / r$ behavior of the Coulomb potential. If the liberated electron is influenced by other orbitals the interaction is called interchannel coupling [45]. The importance of interchannel coupling for HHG has been shown for xenon, where a clear signature of the giant dipole resonance of the $4 d$ subshell [45] known from photoionization studies has been directly observed in the HHG spectrum of xenon [42]. The theoretical model we are utilizing to capture these aspects is based on a time-dependent configuration-interaction singles (TDCIS) approach [46,47]. We have demonstrated in previous works that this TDCIS approach is ideal to study systematically multichannel effects in situations involving ionization $[48,49]$.

The paper is organized as follows. In Sec. II, we give an overview of our theoretical method, which we use to 
systematically study the influence of various approximations of the residual electron-ion interaction on the HHG spectrum. In Sec. III, we explain the system parameters used in our calculations. The results are discussed in detail in Sec. IV. Atomic units [50] are employed throughout, unless otherwise noted.

\section{THEORETICAL METHODS}

The time-dependent Schrödinger equation of an $\mathrm{N}$-electron system exposed to a linearly polarized external electric field is given by

$$
\begin{gathered}
i \frac{\partial}{\partial t}|\Psi(t)\rangle=\hat{H}(t)|\Psi(t)\rangle, \\
\hat{H}(t)=\hat{H}_{0}+\hat{H}_{1}-E(t) \hat{z},
\end{gathered}
$$

where $|\Psi(t)\rangle$ is the full $N$-electron wave function and $\hat{H}(t)$ is the exact $N$-body Hamiltonian, which can be partitioned into three main parts: (1) $\hat{H}_{0}=\hat{F}-i \eta \hat{W}$ is the sum of the time-independent Fock operator $\hat{F}$ and a complex absorbing potential (CAP), where $\left\langle\mathbf{x}|\hat{W}| \mathbf{x}^{\prime}\right\rangle=\left[r-r_{\mathrm{CAP}}\right]^{2} \Theta(r-$ $\left.r_{\mathrm{CAP}}\right) \delta\left(\mathbf{x}-\mathbf{x}^{\prime}\right)$ and $\Theta(r)$ is the Heaviside step function; (2) the electron-electron interactions that cannot be described by the mean-field potential in $\hat{H}_{0}$ are captured by $\hat{H}_{1}$ (= $\hat{V}_{C}-\hat{V}_{\mathrm{HF}}-E_{\mathrm{HF}}$; for a detailed description of these quantities, see Ref. [47]); (3) the term $E(t) \hat{z}$ is the laser-matter interaction in the electric dipole approximation. The CAP serves a purely numerical purpose. It prevents artificial reflections of the ionized photoelectron from the radial grid boundary and is located far away from the atom. This is controlled by the parameter $r_{\mathrm{CAP}}$.

Solving numerically the full $N$-electron system is currently out of reach without making any approximations to the Hamiltonian or the wave function. In strong-field processes such as HHG, Eq. (1a) is commonly reduced to an effective one-electron system, where only one electron of the outermost valence shell is allowed to respond to the electric field and all other electrons are frozen or completely neglected. Here, we take an alternative way, by describing the full $N$-electron wave function and making no approximations to the Hamiltonian. Specifically, we use the configuration-interaction language, where we assume the field-free ground state is well captured by the Hartree-Fock ground state $\left|\Phi_{0}\right\rangle$. We consider only singly excited 1-particle-1-hole configurations ( $1 p 1 h$ configurations) $\left|\Phi_{i}^{a}\right\rangle$. The corresponding TDCIS $N$-electron wave function reads

$$
\begin{aligned}
|\Psi(t)\rangle & =\alpha_{0}(t)\left|\Phi_{0}\right\rangle+\sum_{i, a} \alpha_{i}^{a}(t)\left|\Phi_{i}^{a}\right\rangle, \\
\left|\Phi_{i}^{a}\right\rangle & =\frac{1}{\sqrt{2}}\left(\hat{c}_{a, \uparrow}^{\dagger} \hat{c}_{i, \uparrow}+\hat{c}_{a, \downarrow}^{\dagger} \hat{c}_{i, \downarrow}\right)\left|\Phi_{0}\right\rangle,
\end{aligned}
$$

where $i, j$, and $a, b$ refer to occupied orbitals and unoccupied (virtual) orbitals, respectively, in the Hartree-Fock ground state $\left|\Phi_{0}\right\rangle$. The operators $\hat{c}_{a, \sigma}^{\dagger}$ and $\hat{c}_{a, \sigma}$ create and annihilate, respectively, an electron in the orbital $a$ with spin $\sigma$. By restricting our wave function to $1 p 1 h$ configurations, the interaction captured by $\hat{H}_{1}$ is the residual electron-ion interaction. The equations of motion for the expansion coefficients $\alpha_{0}(t)$ and $\alpha_{i}^{a}(t)$ read

$$
\begin{aligned}
i \dot{\alpha}_{0}(t)= & -E(t) \sum_{i, a}\left(\Phi_{0}|\hat{z}| \Phi_{i}^{a}\right) \alpha_{i}^{a}(t) \\
i \dot{\alpha}_{i}^{a}(t)= & \left(\epsilon_{a}-\epsilon_{i}\right) \alpha_{i}^{a}(t)+\sum_{b, j}\left(\Phi_{i}^{a}\left|\hat{H}_{1}\right| \Phi_{j}^{b}\right) \alpha_{j}^{b}(t) \\
& -E(t)\left(\left(\Phi_{i}^{a}|\hat{z}| \Phi_{0}\right) \alpha_{0}(t)+\sum_{b, j}\left(\Phi_{i}^{a}|\hat{z}| \Phi_{j}^{b}\right) \alpha_{j}^{b}(t)\right)
\end{aligned}
$$

where $\epsilon_{p}$ are the orbital energies of the orbitals $\left|\varphi_{p}\right\rangle$, which are eigenstates of the time-independent Fock operator (i.e., $\hat{H}_{0}\left|\varphi_{p}\right\rangle=\epsilon_{p}\left|\varphi_{p}\right\rangle$ ). The expression (| stands for a dual vector with respect to the symmetric inner product [i.e., $\left(\varphi_{p} \mid \varphi_{q}\right)=$ $\left.\delta_{p, q}\right]$, which differs from the Hermitian inner product. A detailed description of our implementation of the TDCIS method can be found in Ref. [47].

The exact treatment of the residual electron-ion interaction is numerically very demanding. In order to be able to treat the full electron-ion interaction, we are exploiting as much symmetry as possible. We have already used one symmetry with Eq. (2b) [i.e., the total spin of the system $(S=0)$ is conserved]. The second symmetry we are exploiting arises from the restriction to linearly polarized pulses and benefits us in two ways. Firstly, the orbital-angular-momentum projection $m_{a}$ of the excited electron and the orbital-angular-momentum projection $m_{i}$ of the hole state must be the same for each $\left|\Phi_{i}^{a}\right\rangle$. Secondly, the coefficients $\alpha_{i}^{a}(t)$ are the same whether an electron with orbital-angular-momentum projection $m$ or $-m$ is excited. As a result, only the gerade parity configurations $\left|\Phi_{i}^{a}\right\rangle_{g}$ need to be considered because ungerade parity configurations $\left|\Phi_{i}^{a}\right\rangle_{u}$ will not be populated due this symmetry. The gerade and ungerade parity configurations are defined as

$$
\left|\Phi_{i}^{a}\right\rangle_{g / u}:=\frac{1}{\sqrt{2}}\left(\left|\Phi_{+i}^{+a}\right\rangle \pm\left|\Phi_{-i}^{-a}\right\rangle\right)
$$

where the orbital indices $\pm a$ and $\pm i$ stand for triplets of quantum numbers $(n, l, \pm m)$ with $n$ being the radial quantum number, $l$ being the orbital angular momentum, and $\pm m$ being the orbital-angular-momentum projection. The configuration $\left|\Phi_{i}^{a}\right\rangle$ with $m_{i}=m_{a}=0$ is a special case and has gerade parity. Since, as already mentioned, for linearly polarized light $\left|\Phi_{i}^{a}\right\rangle_{u}$ will not be populated and only $\left|\Phi_{i}^{a}\right\rangle_{g}$ needs to be considered, we drop the index $g$ such that $\left|\Phi_{i}^{a}\right\rangle$ refers to gerade parity configurations from now on. The matrix elements for the gerade parity configurations as they appear in Eq. (3) are given by

$$
\begin{aligned}
& \left(\Phi_{i}^{a}|\hat{z}| \Phi_{j}^{b}\right)=z_{(+a,+b)} \delta_{i, j}-z_{(+j,+i)} \delta_{a, b}, \\
& \left(\Phi_{0}|\hat{z}| \Phi_{i}^{a}\right)=z_{(+i,+a)} \begin{cases}\sqrt{2}, & m_{a}=m_{i}=0 \\
2, & m_{a}=m_{i} \neq 0 \\
0, & m_{a} \neq m_{i},\end{cases}
\end{aligned}
$$




$$
\begin{aligned}
& \left(\Phi_{i}^{a}\left|\hat{H}_{1}\right| \Phi_{j}^{b}\right) \\
& =\left(4 v_{(+a,+j ;+i,+b)}-v_{(+a,+j ;+b,+i)}-v_{(+a,-j ;-b ;+i)}\right) \\
& \quad \times \begin{cases}1, & m_{i} \neq 0 \neq m_{j} \\
\frac{1}{2}, & m_{i}=0=m_{j} \\
\sqrt{2}^{-1}, & \text { otherwise }\end{cases}
\end{aligned}
$$

where we made use of the symmetries $z_{(+a,+i)}=$ $z_{(-a,-i)}, v_{+a,+j ;+b,+i}=v_{-a,-j ;-b,-i}$ and $v_{+a,+j ;+i,+b}=$ $v_{-a,-j ;-i,-b}=v_{+a,-j ;+i,-b}$. In the last equation the relations $m_{a}=m_{i}$ and $m_{b}=m_{j}$ are used, which holds only in the case of linearly polarized light. The round parentheses in the indices of the matrix elements indicate the symmetric inner product mentioned above [47].

In the following, we study in detail three scenarios for $\hat{H}_{1}$ : (1) no approximation is made and the residual Coulomb interaction is treated exactly within the CIS configuration space, (2) only intrachannel interactions $\left[\left(\Phi_{i}^{a}\left|\hat{H}_{1}\right| \Phi_{j}^{b}\right)=0\right.$ if $i \neq j$ ] are considered, (3) a symmetrized version of the intrachannel interaction is used such that the angular momentum of the excited electron cannot be changed, thus simulating a spherically symmetric ion potential. When only intrachannel interactions are allowed, different orbitals will behave almost independently. Only via the ground-state depopulation can they indirectly influence each other. The symmetrization in model (3) is done by averaging over all hole states within each $(n, l)$ subshell such that the excited electron sees only a spherically symmetric ion. The symmetrized matrix elements read

$$
\begin{aligned}
v_{(a, i ; b, i)}^{\mathrm{symm}} & :=\frac{1}{2 l_{i}+1} \sum_{m_{i}} v_{(a, i ; b, i)}, \\
v_{(a, i ; i, b)}^{\mathrm{symm}} & :=\frac{1}{2 l_{i}+1} \sum_{m_{i}} v_{(a, i ; i, b)},
\end{aligned}
$$

where in Eq. (6b) we additionally set $m_{a}=m_{i}=m_{b}$ before we perform the sum. This step can be justified, since we are using linearly polarized light and our model can only have 1-particle1-hole configurations with $m_{a}=m_{i}$. In both cases, one finds that the symmetrized matrix elements are proportional to $\delta_{l_{a}, l_{b}}$ and $\delta_{m_{a}, m_{b}}$.

The HHG spectrum, which is calculated via the expectation value of the electric dipole moment $\langle z\rangle(t)$, reads [51,52]

$$
S(\omega)=\frac{1}{20} \frac{1}{3 \pi c^{3}}\left|\int_{-\infty}^{\infty} d t\left[\frac{d^{2}}{d t^{2}}\langle z\rangle(t)\right] e^{-i \omega t}\right|^{2} .
$$

Next to the HHG spectrum, we will focus our discussion also on the hole populations $\rho_{i}(t)$ generated during the HHG process. These populations are calculated with the help of the ion density matrix, which is described in detail in Ref. [47]. The ground-state population is given by $\rho_{0}(t)=\left|\alpha_{0}(t)\right|^{2}$.
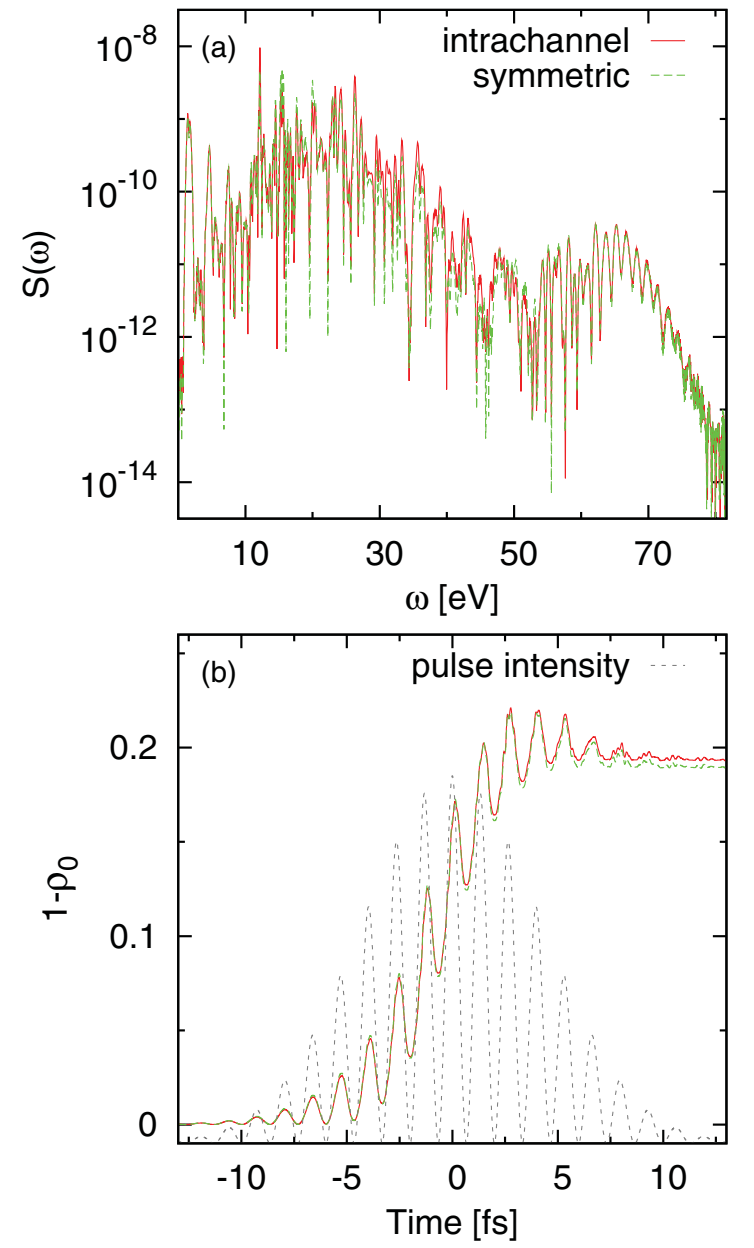

FIG. 1. (Color online) The HHG spectra (a) and the depopulations of the ground state (b) of argon are compared for the intrachannel approximation (red solid line) and for the symmetrized intrachannel approximation (green dashed line). In both cases only $3 p_{0}$ (singlechannel) is active. The intensity profile of the pulse is shown in (b). The pulse parameters are as follows: $E_{\max }=0.085, \omega=$ $0.057(\approx 800 \mathrm{~nm})$, and $\tau=413(\approx 10 \mathrm{fs})$.

\section{NUMERICAL DISCUSSION}

All presented results were calculated with the XCID package, ${ }^{1}$ which makes explicit use of the symmetries discussed in Sec. II. All argon calculations presented in Sec. IV were done for a laser pulse with a peak field strength of $E_{\max }=0.085$, a carrier frequency $\omega=0.057(\approx 800 \mathrm{~nm})$, and a full width at half maximum (FWHM) pulse duration of $\tau=413(\approx 10 \mathrm{fs})$. The classical turning radius of the electron for such a pulse is $r_{\mathrm{HHG}}=E_{\mathrm{max}} / \omega^{2} \approx 26$. As described in Ref. [47], we use a nonuniform grid with the mapping function,

$$
r(x)=r_{\max } \frac{\zeta}{2} \frac{1+x}{1-x+\zeta}, \quad x \in[-1,1]
$$

\footnotetext{
${ }^{1}$ S. Pabst, L. Greenman, and R. Santra, XCID program package for multichannel ionization dynamics, DESY, Hamburg, Germany, 2011, Rev. 481, with contributions from P. J. Ho.
} 
All calculations were done with a radial grid radius $r_{\max }=120$, 480 radial grid points, and mapping parameter $\zeta=1.0$. The CAP starts at a radius $r_{\mathrm{CAP}}=100$ and has a strength $\eta=0.01$. The maximum angular momentum employed was $l_{\max }=80$. Furthermore, we find that for excited electrons with an orbital angular momentum $l>6$ the multipole terms with $L_{c}>0$ are negligibly small and the dominant $\hat{H}_{1}$ contribution comes from the monopole term. It is, therefore, a good approximation to consider only the monopole term of $\hat{H}_{1}$ when any orbital angular momentum of the involved orbitals is larger than 6 [for details see Ref. [47]].

\section{RESULTS}

We begin our discussion with the single-channel model by allowing only the $3 p_{0}$ orbital to be active. In Fig. 1, we compare the HHG spectra and the depopulations of the ground state of argon for a spherically symmetric electron-ion interaction (labeled symmetric) with the exact electron-ion interaction (labeled intrachannel). Note, interchannel contributions do not exist in a single-channel model. The spherically symmetric $\hat{H}_{1}$ has no tensorial multipole moments besides a monopole term, since the angular momentum of the electron cannot be changed. In the intrachannel and interchannel models all multipole contributions are included in $\hat{H}_{1}$. The depopulations [shown in Fig. 1(b)] show only small deviations during and after the pulse. The final depopulation probabilities are almost identical. Similarly, the HHG spectra [see Fig. 1(a)] show only small differences in the energy region of $30-50 \mathrm{eV}$, where also the photoionization cross sections (not shown) differ by up to $30 \%$ from each other. The Cooper minimum in the HHG spectra can be reproduced and lies between 40 and $50 \mathrm{eV}$. The low curvature of the shape of the Cooper minimum prevents a more precise localization of the minimum.

Due to the costly treatment of the residual electron-ion interaction it is common to reduce an HHG calculation to a one-electron calculation, where the electron moves in a local, spherically symmetric model potential, which describes the correct behavior for short and long distances of the electron-ion interaction and reproduces the ionization potential. This is the SAE approach, which is in spirit very close to our singlechannel model with a spherically symmetric $\hat{H}_{1}$ (green dashed line in Fig. 1). However, there exists one major difference to typical SAE calculations. In the SAE approach one only describes one electron, which can move freely everywhere on the pseudopotential surface, and does not fulfill the Pauli principle, meaning there is no mechanism in this approach that can prevent the electron to move into orbitals that are already occupied by the $N-1$ frozen electrons. Enforcing the Pauli principle is critical for the one-electron reduced density matrix to be $N$-representable, that is, to represent a realistic $N$-electron system $[53,54]$. In our theory, we describe always the entire $N$ electron wave function and due to the anticommutator relation of the creation and annihilation operators in Eq. (2b) the Pauli principle is ensured at all times. Recent works for molecular systems have pointed out the importance of the Pauli principle particularly for tomographic purposes $[55,56]$.
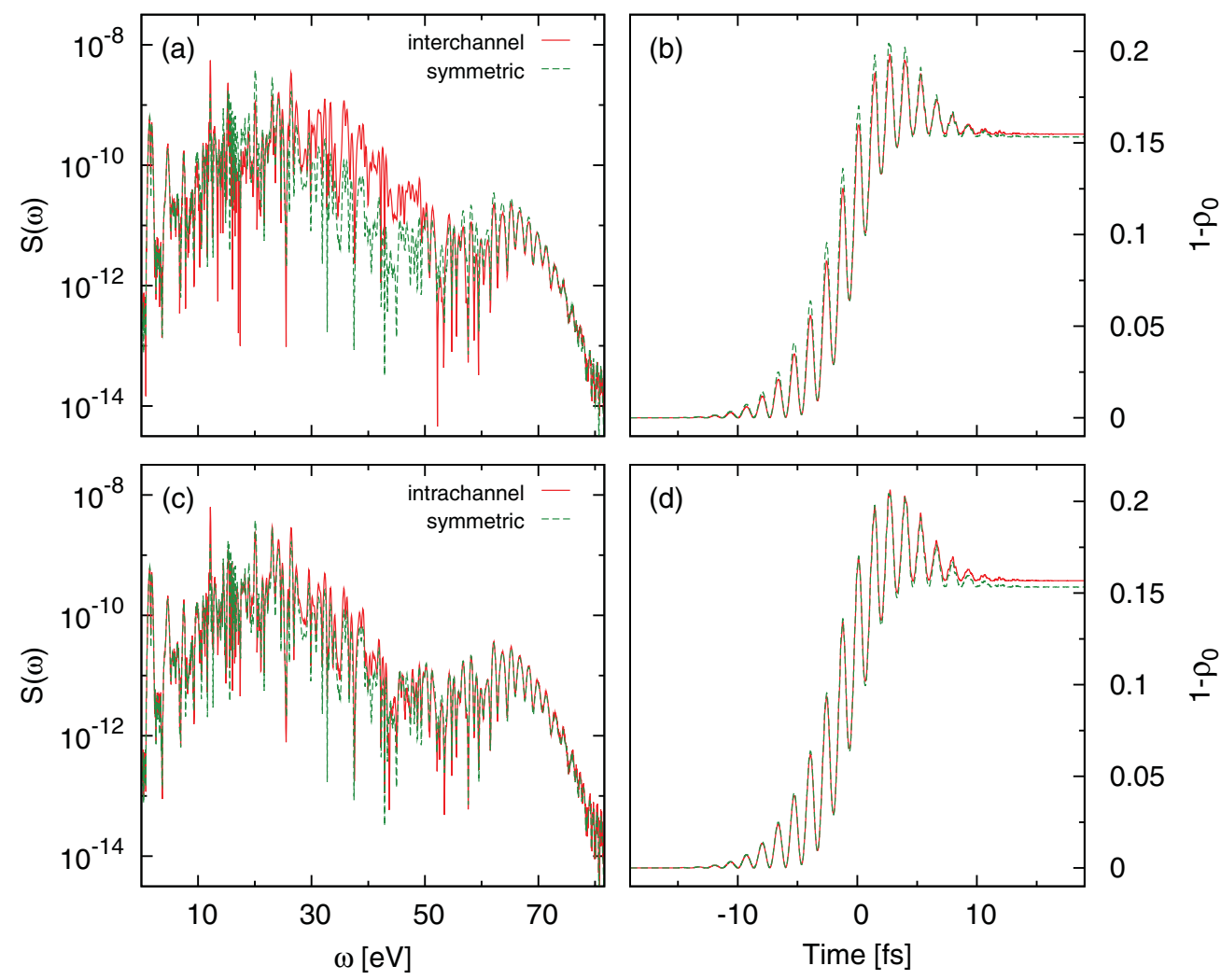

FIG. 2. (Color online) The HHG spectra (a) and (c) and the depopulations of the ground state (b) and (d) of argon are shown for $3 s$ and all $3 p$ orbitals active. In (a) and (b) the interchannel and in (c) and (d) the intrachannel approximation is compared with the symmetrized intrachannel approximation. The pulse parameters are the same as in Fig. 1. 

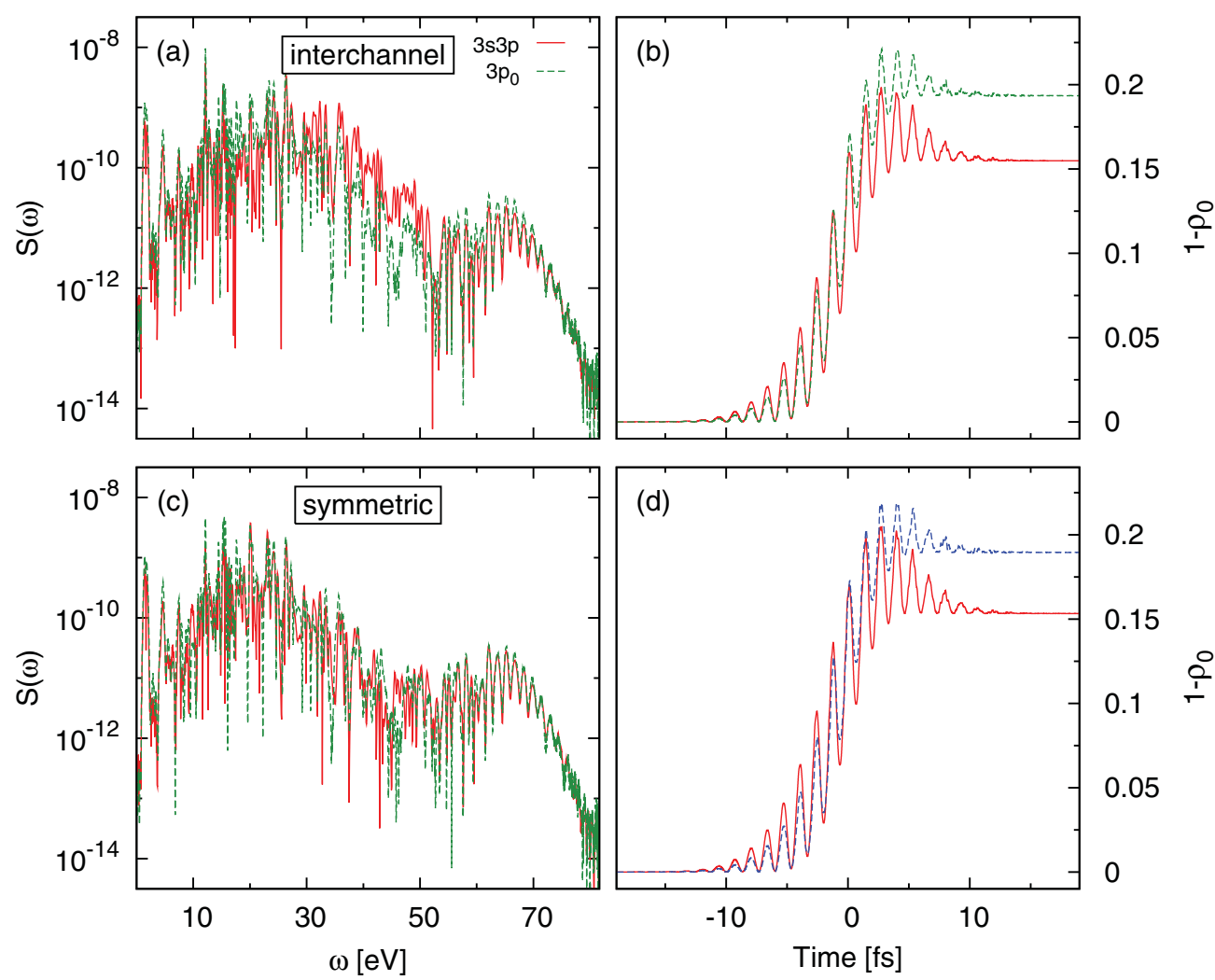

FIG. 3. (Color online) The HHG spectra [(a) and (c)] and the depopulations of the ground state [(b) and (d)] of argon are shown. The single-channel (only $3 p_{0}$ active) calculation is compared with the multichannel calculation ( $3 s$ and all $3 p$ active). No approximation $[($ a) and (b)] and the symmetrized intrachannel approximation [(c) and (d)] are made to the electron-ion interaction. The pulse parameters are the same as in Fig. 1.

Now we consider the impact of the approximation of the residual electron-ion interaction in the multichannel scenario, where we allow also $3 p_{ \pm 1}$ electrons and the $3 s$ electrons to get ionized. All differences seen in Figs. 2, 3, and 4 originate solely from physics within the $3 p$ manifold. The impact of the $3 s$ orbital is rather small on the HHG spectra as well as on the depopulation of the ground state. That is not a surprise due to the high $3 s$ ionization potential, which is around $18 \mathrm{eV}$ higher than the ionization potential of the $3 p$ orbitals. The hole population of $3 s$ (not shown) is over 100 times smaller than the hole populations in the $3 p$ shell.

In Fig. 2 the HHG spectra and depopulations are shown for different approximations of $\hat{H}_{1}$. The simplification to a spherically symmetric potential [see Figs. 2(a) and 2(b)] underestimates the HHG spectrum by up to two orders of magnitude in the energy region of 30-50 eV. In addition, the shape of the Cooper minimum has now drastically changed. The position of the Cooper minimum is much more clearly defined in the interchannel case and lies slightly above $50 \mathrm{eV}$ as found in recent experiments [37,43,44]. For the symmetrized intrachannel approximation, the Cooper minimum lies between 40 and $50 \mathrm{eV}$, similar to the single-channel results. The depopulation dynamics is not affected by the approximation of the electron-ion interaction. In Figs. 2(c) and 2(d) the results of the intrachannel calculation are compared with the results obtained from the symmetrized intrachannel approximation. The relative differences between these two models never exceed a factor larger than 2 and are confined to the energy region of $30-50 \mathrm{eV}$. The origin of these differences is the lack of multipole effects in the symmetrized intrachannel model. In comparison to the interchannel results, the multipole effects are much smaller than the interchannel effects seen in Fig. 2(a).

The photon energy range of $30-50 \mathrm{eV}$ corresponds to a recollision electron energy range of $15-35 \mathrm{eV}$ and a de Broglie wavelength of 2-3 $\AA$. It seems that electrons with these wavelengths are most sensitive to the exact residual ion-electron interaction and, therefore, simplifications of the interaction become most evident in the corresponding photon energy regime. In the same energy region the photoionization cross section is most sensitive to the approximation made to the electron-ion interaction [45]. Our calculations (not shown) confirm that the differences in the cross sections between the intrachannel and symmetrized intrachannel model are quite small, whereas the differences to the interchannel model are up to one order of magnitude larger (and can reach values up to $20 \mathrm{Mb}$ ). The fact that the photoionization cross sections and the HHG spectra behave similarly (for different approximations to the electron-ion interaction) supports the picture that HHG has a close connection to photoionization. In the limit that the electron de Broglie wavelength is much longer or shorter than the characteristic length scale of the residual electron-ion interaction, the results do not depend on the detailed structure of electron-ion interaction. This may explain why the HHG 


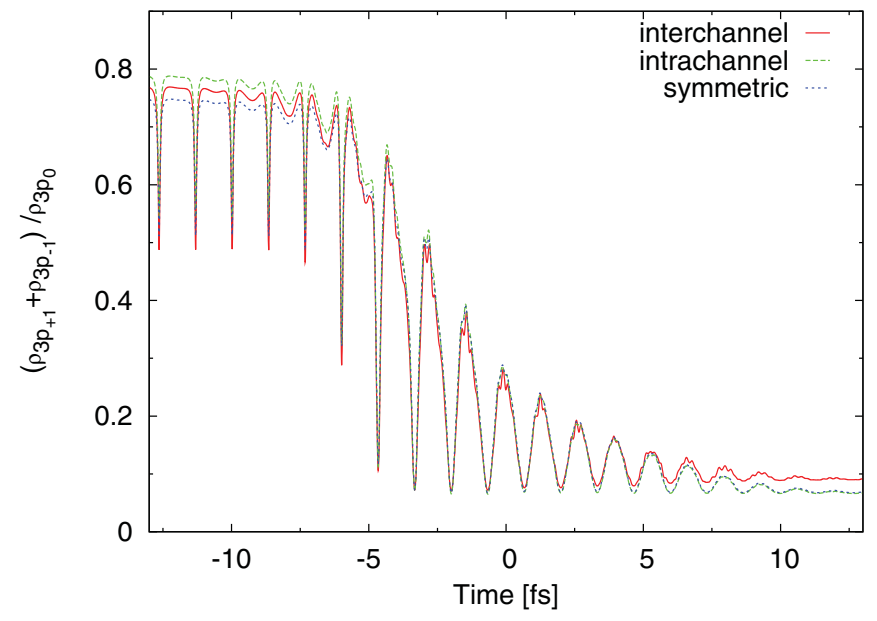

FIG. 4. (Color online) The ratio of the hole populations $\rho_{3 p_{1}}+$ $\rho_{3 p_{-1}}$ and $\rho_{3 p_{0}}$ is shown for different approximations to the electronion interaction. The pulse parameters are the same as in Fig. 1.

spectrum does not alter significantly for photon energies smaller than $20 \mathrm{eV}$ and photon energies close to the cutoff region.

We have seen that approximations to the electron-ion interaction can cause differences in the HHG yield, particularly, when multiple orbitals are considered. Not all electron-ion approximations make the HHG spectrum sensitive to whether a single orbital or multiple orbitals are active. In Fig. 3 we compare the HHG spectrum and the depopulation of the ground state for a single-channel (only $3 p_{0}$ active) and for a multichannel (all $3 p$ and $3 s$ are active) calculations. Figures $3(\mathrm{a})$ and $3(\mathrm{~b})$ are calculated with the exact $\hat{H}_{1}$ term including interchannel and multipole effects in the residual electron-ion interaction. The HHG signal strength for the single-channel calculation is strongly reduced in the spectral range of $30-50 \mathrm{eV}$, whereas the signal is slightly enhanced in the cutoff region. When the symmetrized intrachannel approximation is made [see Figs. 3(c) and 3(d)], the HHG spectra are almost identical whether single-channel or multichannel calculations are performed. This stands in contrast to the interchannel results, where the interchannel coupling causes strong differences in the HHG yield. The depopulation is overestimated by $\approx 25 \%$ regardless of the approximation made to the electron-ion interaction.

We have seen the strong differences in the HHG spectra and in the depopulations depending on whether only $3 p_{0}$ or all $3 p$ electrons are active (the contributions from $3 s$ are negligible small). Does that also mean the ionization probabilities of $3 p_{ \pm 1}$ are comparable with $3 p_{0}$ ? The ratio $\left(\rho_{3 p_{1}}+\rho_{3 p_{-1}}\right) / \rho_{3 p_{0}}$ is shown in Fig. 4 for the different models. Note that for linearly polarized light $\rho_{3 p_{+1}}=\rho_{3 p_{-1}}$. Before the pulse, all ratios are close to 0.8 and drop to $\approx 0.1$ after the pulse. Both intrachannel models lead even to the same final ratio. The small ratios after the pulse show that, at least ultimately, mainly the $3 p_{0}$ orbital gets ionized, which is at the heart of the SAE approximation. The oscillations in the ratios during the pulse are in phase with the oscillations in the electric field. They are a direct consequence of the projection of the wave function onto the field-free states in the presence of the laser field. In contrast to the HHG spectra (see Fig. 2), the effects of the intrachannel or symmetric approximation on the population dynamics are quite small.

\section{CONCLUSION}

We have described the HHG process with a many-body approach, namely TDCIS, where we describe the entire $N$ electron wave function. This allows us to fulfill the Pauli principle at all times. Our results show that multichannel effects in the residual electron-ion interaction, which is a combination of the bare nuclear potential and the electron-electron interaction for many-electron systems, have a significant influence on the HHG spectrum. They cannot generally be neglected for atoms and specifically not for molecules as recent experiments have shown $[40,41]$. We have demonstrated that orbitals, despite their relatively low ionization probability by the end of the pulse, can lead to surprisingly large modifications of up to 2 orders of magnitude in the HHG spectrum (especially in the energy region of 30-50 eV). While we confirm that after the end of the pulse, the populations of the $3 p_{ \pm 1}$ orbitals are relatively small, their contributions during the pulse are not small and have indirectly through interchannel coupling a significant impact on the HHG yield.

We saw that neglecting interchannel interactions leads to large changes in the HHG yield. Multipole effects influence the spectra but not as dramatically as interchannel effects do. All deviations in the HHG yield are in the $30-50 \mathrm{eV}$ energy region, which corresponds to a de Broglie wavelength of the recolliding electron between 2-3 $\AA$. This coincides with the characteristic length scale on which the electron-ion interaction goes over into a pure long-range $1 / r$ potential. In contrast to the large disagreement in the HHG spectra between the single-channel and multichannel calculations including interchannel interactions we found that by using the symmetric interaction the HHG spectra look quite the same whether or not a single or multiple channels participate in the HHG process. This comparison directly shows that the population of an orbital does not map directly to its importance in the HHG mechanism.

All these observations demonstrate that many-body effects enter in the HHG spectrum and need to be understood in order to successfully use them for tomographic imaging [12]. The time-dependent configuration-interaction approach provides a clear pathway how these and higher-order effects can be taken into account. Recent works [57] have suggested that multielectron excitations are not a dominant factor. All essential multielectron effects can be captured by single-electron excitations including interchannel interactions. This makes the TDCIS approach perfectly suited for studying many-body effects in HHG.

\section{ACKNOWLEDGMENTS}

This work has been supported by the Deutsche Forschungsgemeinschaft (DFG) under Grant No. SFB 925/A5. We thank Sang-Kil Son for helpful discussions. 
[1] M. Hentschel, R. Kienberger, C. Spielmann, G. A. Reider, N. Milosevic, T. Brabec, P. Corkum, U. Heinzmann, M. Drescher, and F. Krausz, Nature (London) 414, 509 (2001).

[2] G. Sansone, F. Kelkensberg, J. F. Perez-Torres, F. Morales, M. F. Kling, W. Siu, O. Ghafur, P. Johnsson, M. Swoboda, E. Benedetti, F. Ferrari, F. Lepine, J. L. Sanz-Vicario, S. Zherebtsov, I. Znakovskaya, A. L'Huillier, M. Yu. Ivanov, M. Nisoli, F. Martin, and M. J. J. Vrakking, Nature (London) 465, 763 (2010).

[3] T. Sekikawa, A. Kosuge, T. Kanai, and S. Watanabe, Nature (London) 432, 605 (2004).

[4] N. A. Papadogiannis, B. Witzel, C. Kalpouzos, and D. Charalambidis, Phys. Rev. Lett. 83, 4289 (1999).

[5] P. M. Paul, E. S. Toma, P. Breger, G. Mullot, F. Aug, P. Balcou, H. G. Muller, and P. Agostini, Science 292, 1689 (2001).

[6] K. P. Singh, F. He, P. Ranitovic, W. Cao, S. De, D. Ray, S. Chen, U. Thumm, A. Becker, M. M. Murnane, H. C. Kapteyn, I. V. Litvinyuk, and C. L.Cocke, Phys. Rev. Lett. 104, 023001 (2010).

[7] F. Krausz and M. Ivanov, Rev. Mod. Phys. 81, 163 (2009).

[8] P. H. Bucksbaum, Science 317, 766 (2007).

[9] E. A. Gibson, A. Paul, N. Wagner, R. Tobey, S. Backus, I. P. Christov, M. M. Murnane, and H. C. Kapteyn, Phys. Rev. Lett. 92, 033001 (2004).

[10] M. Uiberacker, T. Uphues, M. Schultze, A. J. Verhoef, V. Yakovlev, M. F. Kling, J. Rauschenberger, N. M. Kabachnik, H. Schroder, M. Lezius, K. L. Kompa, H.-G. Muller, M. J. J. Vrakking, S. Hendel, U. Kleineberg, U. Heinzmann, M. Drescher, and F. Krausz, Nature (London) 446, 627 (2007).

[11] A. L. Lytle, X. Zhang, J. Peatross, M. M. Murnane, H. C. Kapteyn, and O. Cohen, Phys. Rev. Lett. 98, 123904 (2007).

[12] J. Itatani, J. Levesque, D. Zeidler, H. Niikura, H. Pepin, J. Kieffer, P. Corkum, and D. Villeneuve, Nature (London) 432, 867 (2004).

[13] S. Baker, J. S. Robinson, C. A. Haworth, H. Teng, R. A. Smith, C. C. Chiril, M. Lein, J. W. G. Tisch, and J. P. Marangos, Science 312, 424 (2006).

[14] A. L. Cavalieri, N. Mueller, T. Uphues, V. S. Yakovlev, A. Baltuska, B. Horvath, B. Schmidt, L. Bluemel, R. Holzwarth, S. Hendel, M. Drescher, U. Kleineberg, P. M. Echenique, R. Kienberger, F. Krausz, and U. Heinzmann, Nature (London) 449, 1029 (2007).

[15] D. Shafir, Y. Mairesse, H. J. Wrner, K. Rupnik, D. M. Villeneuve, P. B. Corkum, and N. Dudovich, New J. Phys. 12, 073032 (2010).

[16] M. Drescher, M. Hentschel, R. Kienberger, M. Uiberacker, V. Yakovlev, A. Scrinzi, T. Westerwalbesloh, U. Kleineberg, U. Heinzmann, and F. Krausz, Nature (London) 419, 803 (2002).

[17] H. J. Woerner, J. B. Bertrand, D. V. Kartashov, P. B. Corkum, and D. M. Villeneuve, Nature (London) 466, 604 (2010).

[18] E. Goulielmakis, Z.-H. Loh, A. Wirth, R. Santra, N. Rohringer, V. S. Yakovlev, S. Zherebtsov, T. Pfeifer, A. M. Azzeer, M. F. Kling, S. R. Leone, and F. Krausz, Nature (London) 466, 739 (2010).

[19] A. Wirth, M. Th. Hassan, I. Grguraš, J. Gagnon, A. Moulet, T. T. Luu, S. Pabst, R. Santra, Z. A. Alahmed, A. M. Azzeer, V. S. Yakovlev, V. Pervak, F. Krausz, and E. Goulielmakis, Science 334, 195 (2011).

[20] M. Lewenstein, P. Balcou, M. Y. Ivanov, A. L'Huillier, and P. B. Corkum, Phys. Rev. A 49, 2117 (1994).

[21] P. B. Corkum, Phys. Rev. Lett. 71, 1994 (1993).
[22] K. J. Schafer, B. Yang, L. F. DiMauro, and K. C. Kulander, Phys. Rev. Lett. 70, 1599 (1993).

[23] A. Gordon, F. X. Kärtner, N. Rohringer, and R. Santra, Phys. Rev. Lett. 96, 223902 (2006).

[24] V.-M. Gkortsas, S. Bhardwaj, E. L. Falco-Filho, K.-H. Hong, A. Gordon, and F. X. Krtner, J. Phys. B 44, 045601 (2011).

[25] A.-T. Le, R. R. Lucchese, S. Tonzani, T. Morishita, and C. D. Lin, Phys. Rev. A 80, 013401 (2009).

[26] L. Landau and E. Lifschitz, Quantum Mechanics NonRelativistic (Pergamon Press, New York, 1965).

[27] C. D. Lin, A.-T. Le, Z. Chen, T. Morishita, and R. Lucchese, J. Phys. B 43, 122001 (2010).

[28] T. Morishita, A.-T. Le, Z. Chen, and C. D. Lin, Phys. Rev. Lett. 100, 013903 (2008).

[29] C. Vozzi, F. Calegari, E. Benedetti, J.-P. Caumes, G. Sansone, S. Stagira, M. Nisoli, R. Torres, E. Heesel, N. Kajumba, J. P. Marangos, C. Altucci, and R. Velotta, Phys. Rev. Lett. 95, 153902 (2005).

[30] M. Y. Emelin, M. Y. Ryabikin, and A. M. Sergeev, New J. Phys. 10, 025026 (2008).

[31] W. Boutu, S. Haessler, H. Merdji, P. Breger, G. Waters, M. Stankiewicz, L. J. Frasinski, R. Taieb, J. Caillat, A. Maquet, P. Monchicourt, B. Carre, and P. Salieres, Nat. Phys. 4, 545 (2008).

[32] B. A. Sickmiller and R. R. Jones, Phys. Rev. A 80, 031802 (2009).

[33] J. Z. Kamiński and F. Ehlotzky, Phys. Rev. A 54, 3678 (1996).

[34] O. Smirnova, A. S. Mouritzen, S. Patchkovskii, and M. Y. Ivanov, J. Phys. B 40, F197 (2007).

[35] A. Abdurrouf and F. H. M. Faisal, Phys. Rev. A 79, 023405 (2009).

[36] K. C. Kulander, K. J. Schafer, and J. L. Krause, Int. J. Quantum Chem. 40, 415 (1991).

[37] J. Higuet, H. Ruf, N. Thiré, R. Cireasa, E. Constant, E. Cormier, D. Descamps, E. Mével, S. Petit, B. Pons, Y. Mairesse, and B. Fabre, Phys. Rev. A 83, 053401 (2011).

[38] M. Awasthi, Y. V. Vanne, A. Saenz, A. Castro, and P. Decleva, Phys. Rev. A 77, 063403 (2008).

[39] I. A. Ivanov and A. S. Kheifets, Phys. Rev. A 79, 053827 (2009).

[40] B. K. McFarland, J. P. Farrell, P. H. Bucksbaum, and M. Ghr, Science 322, 1232 (2008).

[41] O. Smirnova, Y. Mairesse, S. Patchkovskii, N. Dudovich, D. Villeneuve, P. Corkum, and M. Y. Ivanov, Nature (London) 460, 972 (2009).

[42] A. D. Shiner, B. E. Schmidt, C. Trallero-Herrero, H. J. Worner, S. Patchkovskii, P. B. Corkum, J. Kieffer, F. Legare, and D. M. Villeneuve, Nat. Phys. 7, 464 (2011).

[43] H. J. Wörner, H. Niikura, J. B. Bertrand, P. B. Corkum, and D. M. Villeneuve, Phys. Rev. Lett. 102, 103901 (2009).

[44] J. P. Farrell, L. S. Spector, B. K. McFarland, P. H. Bucksbaum, M. Gühr, M. B. Gaarde, and K. J. Schafer, Phys. Rev. A 83, 023420 (2011).

[45] A. F. Starace, in Handbuch der Physik, Vol. 31, edited by W. Mehlhorn (Springer, Berlin, 1980), pp. 1-121.

[46] N. Rohringer, A. Gordon, and R. Santra, Phys. Rev. A 74, 043420 (2006).

[47] L. Greenman, P. J. Ho, S. Pabst, E. Kamarchik, D. A. Mazziotti, and R. Santra, Phys. Rev. A 82, 023406 (2010).

[48] N. Rohringer and R. Santra, Phys. Rev. A 79, 053402 (2009). 
[49] S. Pabst, L. Greenman, P. J. Ho, D. A. Mazziotti, and R. Santra, Phys. Rev. Lett. 106, 053003 (2011).

[50] P. J. Mohr, B. N. Taylor, and D. B. Newell, Rev. Mod. Phys. 80, 633 (2008).

[51] A. Gordon and F. Kärtner, Opt. Express 13, 2941 (2005).

[52] E. Seres, J. Seres, F. Krausz, and C. Spielmann, Phys. Rev. Lett. 92, 163002 (2004).
[53] A. E. Rothman and D. A. Mazziotti, J. Chem. Phys. 132, 104112 (2010).

[54] D. A. Mazziotti, Phys. Rev. Lett. 106, 083001 (2011).

[55] R. Santra and A. Gordon, Phys. Rev. Lett. 96, 073906 (2006).

[56] S. Patchkovskii, Z. Zhao, T. Brabec, and D. M. Villeneuve, Phys. Rev. Lett. 97, 123003 (2006).

[57] S. Sukiasyan, C. McDonald, C. Destefani, M. Y. Ivanov, and T. Brabec, Phys. Rev. Lett. 102, 223002 (2009). 